\title{
An Efficient V2G Optimal Control Method based Price Update Mechanism
}

\author{
Zhang, Wenjie ${ }^{1, a}$, Ma, Zhongjing ${ }^{2, b}$ \\ School of Automation, Beijing Institute of Technology, Beijing 100081, P. R. China \\ E-mail: ${ }^{a}$ zhang_wen_jie521@163.com, ${ }^{b}$ mazhongjing@bit.edu.cn
}

Keywords: plug-in electric vehicles(PEVs); price update mechanism; centralized control; decentralized control

\begin{abstract}
In this paper, we propose a novel decentralized control under price update mechanism for large populations of plug-in electric vehicles(PEVs). We use the PEVs as decentralized power suppliers to supply frequency and voltage amplitude adjustment services to the grid. In the price update mechanism, the PEVs don't need to submit price and maximum power they can be supplied to the grid. For the grid price, the PEVs can make allocation policy decision according to their cost functions and battery capacity. Then the grid update the price by all the PEVs' allocations. Finally we choose the allocation when the price dose not change as the optimal one. The simulation results indicate that the allocations of the price update mechanism converge to the centralized control result.
\end{abstract}

\section{Introduction}

Along with the pollution of the environment and the consumption of non-renewable resources, plug-in electric vehicles are beginning to compete with the conventional petroleum- combustion vehicles and achieve a high speed development. The attendant problem is that a large population of PEVs into grid will make significant impact on the electric grid. The disorderly charging control behavior of the PEVs is disadvantage to the power balance in the grid, even make a severe crash on power system.

Many studies have been undertaken recently to research the potential impacts of large population of PEVs on the power grid [1], [2], [3], [4]. [5] mentions that PEVs will become one of the largest number of grid load in the future and analysis the impact of PEVs' charging behavior at peak hours on the grid.

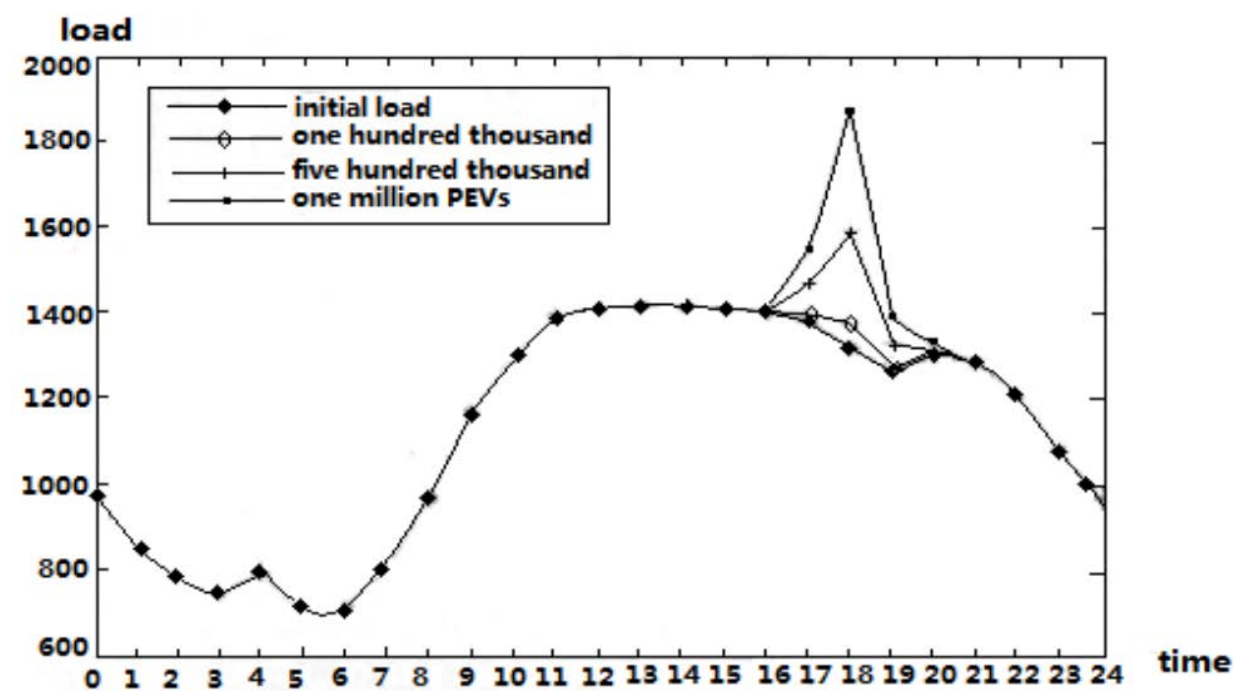

Fig.1 the impact of the disordered PEVs charging on the grid

The charging control of PEVs become more and more important. So much studies are done with charging control of the PEVs. A novel centralized charging control strategy for large populations of PEVs is developed in [6]. A novel decentralized charging control is developed in [7]. Both of the 
centralized and decentralized control strategy achieve Nash equilibrium. [8] proposes a market uniform clearing price(MCP) mechanism to implement the charging problems of plug-in electric vehicles(PEVs) over a finite interval. [9] formulate a decentralized charging algorithm by emulating the charging pattern identified through linear programming optimization solutions, but it also only minimizes the generation costs and this algorithm requires many estimated data.

Many research commitment to service the grid by using the PEVs as the decentralized power. In $[10,11]$, the PEVs participate in secondary frequency regulation , finally both of the owners of PEVs and the grid get benefit. [12,13]presents that the PEVs' active and reactive power could offer service to frequency and voltage regulation. [14] proposes a PSP auction mechanism and converge to a Nash equilibrium.

In this paper, we propose a novel price update mechanism in the sense both of the system welfare and the PEVs welfare. We control the PEVs charging and discharging behaviors under this mechanism to achieve the purpose of frequency and voltage regulation. By this mechanism, the each PEV user could make the allocation according to the demand power and the PEV's cost function. Then the grid collect all the PEV users' allocations and update the electric power price. This process will continue until the price doesn't change. Then this price and allocations are the optimal result for the system.

The paper is organized as follows.

In section 2, we formulate a class of V2G coordination problems for frequency and voltage regulation.

In section 3, we propose a decentralized control method under price update mechanism.

In section 4, we prove that the allocations of decentralized control under price update mechanism converge to centralized control result.

In section 5, we illustrate the decentralized control strategies under price update mechanism could converge to centralized results with numerical examples.

In section 6, we present our conclusion about our proposed decentralized control under price update mechanism.

\section{Formulation of frequency and voltage regulation}

In $[12,13]$, we know that the frequency and voltage can be adjusted by active and reactive power. We donate $P$ and $Q$ as the demand active and reactive power, such that(see in [15])

- $P>0$ indicates that the supply of active power is higher than the demand of active power in the grid; $P<0$ indicates that the supply of active power is lower than the demand of active power in the grid; $Q>0$ indicates that the grid requires compensation of capacitive reactive power;

- $Q<0$ indicates that the grid requires compensation of inductive reactive power.

For demonstration simplicity, in this paper we study the V2G coordination in case $P>0$ and $Q>0$.

We donate $\mathcal{N}$ as the collection of PEVs which participate in the frequency and voltage regulation. Let $u_{n P}$ and $u_{n Q}$ indicate the active power and reactive power of PEV $n$. As we consider above, we have

$$
u_{n P}, \quad u_{n Q}>0
$$

for all $n \in \mathcal{N}$, i.e. each PEV charges and operates as a capacitive load.

We consider the following nominal apparent power constraint for PEV $n$

$$
u_{n P}{ }^{2}+u_{n Q}{ }^{2}=S_{n}{ }^{2} \leq S_{n}^{\max 2}
$$

where $S_{n}^{\max }$ donates the maximum apparent power of the charger PEV $n$.

For active power, we consider a maximum charging rate constraint such that 


$$
u_{n P} \leq u_{n P}^{\max } \triangleq \min \left\{\gamma_{n}, \frac{\Gamma_{n}}{\Delta T}\left(S O C_{\max }-S O C_{n 0}\right)\right\}
$$

where $\gamma_{n}$ donates a safe maximum charging rate, $S O C_{\max }$ and $S O C_{\min }$ represent the minimum and maximum state of charge (SOC) of PEVs, $S O C_{n 0}$ represents the initial SOC value of PEV $n, \Gamma_{n}$ donates the battery energy capacity of PEV $n$ and $\Delta T$ indicates the length of service.

Let $\boldsymbol{u}_{n} \equiv\left(u_{n P}, u_{n Q}\right)$ if $\boldsymbol{u}_{n}$ satisfies the constraints (1)-(3). We donate $g_{n}\left(\boldsymbol{u}_{n}, S O C_{n 0}\right)$ as the local cost function of PEV $n$ and we have

$$
g_{n}\left(u_{n}, S O C_{n 0}\right)=g_{n P}\left(u_{n P}, S O C_{n 0}\right)+g_{n Q}\left(u_{n Q}, S O C_{n 0}\right)
$$

where $g_{n P}\left(u_{n P}, S O C_{n 0}\right)$ and $g_{n Q}\left(u_{n Q}, S O C_{n 0}\right)$ represent the battery cost subject to the charging rates $u_{n P}$ and $u_{n Q}$.

We donate $\boldsymbol{u}_{s} \equiv\left(u_{S P}, u_{s Q}\right)$ as the aggregated strategies of V2G coordination. We have

$$
u_{s P}=\sum_{n=1}^{N} u_{n P}, u_{s Q}=\sum_{n=1}^{N} u_{n Q}
$$

We define a value function of the grid related to $\boldsymbol{u}_{s}$, donate by $v_{s}\left(\boldsymbol{u}_{s} ; P, \mathrm{Q}\right)$, such that

$$
v_{s}\left(\boldsymbol{u}_{s} ; P, Q\right) \triangleq v_{s P}\left(u_{s P} ; P\right)+v_{s Q}\left(u_{s Q} ; Q\right)
$$

where $v_{s P}\left(u_{s P} ; P\right)$ and $v_{s Q}\left(u_{s Q} ; Q\right)$ indicate the grid valuation subject to $u_{s P}$ and $u_{s Q}$.

For notational simplicity, we may consider $g_{n}\left(\boldsymbol{u}_{\boldsymbol{n}}\right) \equiv g_{n}\left(\boldsymbol{u}_{\boldsymbol{n}}, S O C_{n 0}\right), g_{n P}\left(u_{n P}\right) \equiv g_{n P}\left(u_{n P}, S O C_{n 0}\right)$, $g_{n Q}\left(u_{n Q}\right) \equiv g_{n Q}\left(u_{n Q}, S O C_{n 0}\right), v_{s}\left(\boldsymbol{u}_{s}\right) \equiv v_{s}\left(\boldsymbol{u}_{s} ; P, Q\right), v_{s P}\left(u_{s P}\right) \equiv v_{s P}\left(u_{s P} ; P\right)$ and $v_{s Q}\left(u_{s Q}\right) \equiv v_{s Q}\left(u_{s Q} ; Q\right)$.

Let $\mathcal{U}$ denote the collection of power grid strategies and admissible PEV coordination allocations, denoted by $\boldsymbol{u} \equiv\left(\boldsymbol{u}_{s}, \boldsymbol{u}_{n} ; n \in \mathcal{N}\right)$, if $\boldsymbol{u}_{n}$ satisfies (1) - (3).

From (4)-(6), we donate the system valuation function as follows:

$$
J(\boldsymbol{u}) \triangleq v_{s}\left(\boldsymbol{u}_{s}\right)-\sum_{n=1}^{N} g_{n}\left(\boldsymbol{u}_{\boldsymbol{n}}\right)
$$

We also donate the utility function of $\operatorname{PEV} n(n \in \mathcal{N})$, such that

$$
f_{n}\left(\boldsymbol{u}_{\boldsymbol{n}}\right) \triangleq u_{n P} p_{P}+u_{n Q} p_{Q}-g_{n}\left(\boldsymbol{u}_{\boldsymbol{n}}\right)
$$

where $p_{P}$ and $p_{Q}$ indicate the active power price and the reactive power price.

In this paper, we consider the following assumptions:

(A1) $v_{s P}\left(u_{s P}\right)$ and $v_{s Q}\left(u_{s Q}\right)$ are strictly increasing and concave on $u_{s P}$ and $u_{s Q}$, respectively;

(A2) $g_{n P}\left(u_{n P}\right)$ and $g_{n Q}\left(u_{n Q}\right)$, with $n \in \mathcal{N}$, are strictly increasing and convex on $u_{n P}$ and $u_{n Q}$, respectively.

\section{Price update mechanism}

One of the most important part of price update mechanism is the price update formula, such that

$$
\begin{aligned}
& \hat{p}_{P}=p_{P}+\eta_{P}\left(v_{s}^{\prime}\left(\hat{u}_{s P} ; P\right)-p_{P}\right) \\
& \hat{p}_{Q}=p_{Q}+\eta_{Q}\left(v_{s}^{\prime}\left(\hat{u}_{s Q} ; Q\right)-p_{Q}\right)
\end{aligned}
$$

where $\eta_{P}, \eta_{Q} \in(0,1)$. 
PEV $n(n \in \mathcal{N})$ could calculate the optimal allocation for each price $\left(p_{P}, p_{Q}\right)$. To maximum the utility function of PEV $n$, we can get the efficient strategies $\mathcal{U}$. Let $\hat{\boldsymbol{u}}_{n}$ donate the efficient allocation, i.e.

$$
\hat{\boldsymbol{u}}_{n}=\underset{n \in \mathcal{N}, u \in \mathcal{U}}{\arg \max } f_{n}\left(\boldsymbol{u}_{\boldsymbol{n}}\right)
$$

From (9) and (10), we can update the price and get the new allocations.

Then we donate $\varepsilon_{P}$ and $\varepsilon_{Q}$ as price deviation, such that

$$
\begin{aligned}
& \varepsilon_{P}=\left|\hat{p}_{P}-p_{P}\right| \\
& \varepsilon_{Q}=\left|\hat{p}_{Q}-p_{Q}\right|
\end{aligned}
$$

Let a very small positive number $\varepsilon_{\text {stop }}$ donate the end signal. The update process will be end when $\varepsilon_{P} \leq \varepsilon_{\text {stop }}$ and $\varepsilon_{Q} \leq \varepsilon_{\text {stop }}$.

The final allocations should be written as $\boldsymbol{u}_{n}^{*} \equiv\left(u_{n P}^{*}, u_{n Q}^{*}\right), p^{*} \equiv\left(p_{P}^{*}, p_{Q}^{*}\right)$, such that

$$
\begin{aligned}
& \boldsymbol{u}_{n}^{*}=\underset{n \in \mathcal{N}, u \in \mathcal{U}}{\arg \max } f_{n}\left(\boldsymbol{u}_{\boldsymbol{n}}\right) \\
& p_{P}^{*}=v_{S P}^{\prime}\left(u_{s P}^{*}\right) \\
& p_{Q}^{*}=v_{S Q}^{\prime}\left(u_{s Q}^{*}\right)
\end{aligned}
$$

\section{The efficient optimal strategies}

The price update mechanism is a decentralized control method and the allocations under this mechanism are the optimal strategies of the PEVs. We should prove that the strategies are also optimal for the system.

Let $\boldsymbol{u}^{* *}$ donate the optimal allocations of the system, i.e.

$$
\mathbf{u}^{* *}=\underset{u \in \mathcal{U}}{\arg \max } J(\boldsymbol{u})
$$

Lemma 1. The optimal allocations under price update mechanism $\boldsymbol{u}_{n}^{*}$ must satisfy (13).

Proof. The Lagrangian function for the optimization problems (12) subject to (1)-(3) is specified as below

$$
L\left(\boldsymbol{u}_{\boldsymbol{n}}, \lambda_{n P}^{\prime}, \lambda_{n Q}^{\prime}\right)=f_{n}\left(\boldsymbol{u}_{\boldsymbol{n}}\right)+\lambda_{n P}^{\prime}\left(a_{n}-u_{n P}\right)+\lambda_{n S}^{\prime}\left(S_{n}^{\max 2}-u_{n P}^{2}-u_{n Q}^{2}\right)
$$

with $\boldsymbol{u}_{n} \equiv\left(u_{n P}, u_{n Q}\right)$, where $\lambda_{n P}^{\prime}$ is the Lagrange multiplier corresponding to the active power constraint of PEV n, $\lambda_{n S}^{\prime}$ is the Lagrange multiplier corresponding to the apparent power constraint.

The following, together with the equality constraint (5), are the Karush - Kuhn - Tucker (KKT) conditions for the constrained optimisation problems given in (12):

$$
\begin{aligned}
& p_{P}^{*}-g_{n P}^{\prime}\left(u_{n P}^{*}\right)-\lambda_{n P}^{\prime}-2 \lambda_{n S}^{\prime} u_{n P}^{*} \leq 0, u_{n P}^{*} \geq 0, u_{n P}^{*}\left(p_{P}^{*}-g_{n P}^{\prime}\left(u_{n P}^{*}\right)-\lambda_{n P}^{\prime}-2 \lambda_{n S}^{\prime} u_{n P}^{*}\right)=0 \\
& p_{Q}^{*}-g_{n Q}\left(u_{n Q}^{*}\right)-2 \lambda_{n S}^{\prime} u_{n Q}^{*} \leq 0, u_{n Q}^{*} \geq 0, u_{n Q}^{*}\left(p_{Q}^{*}-g_{n Q}^{\prime}\left(u_{n Q}^{*}\right)-2 \lambda_{n S}^{\prime} u_{n Q}^{*}\right)=0 \\
& u_{n P}^{*} \leq a_{n}, \lambda_{n P}^{\prime} \geq 0, \lambda_{n P}^{\prime}\left(a_{n}-u_{n P}^{*}\right)=0
\end{aligned}
$$




$$
u_{n P}^{* 2}+u_{n Q}^{* 2} \leq S_{n}^{\max ^{2}}, \lambda_{n S}^{\prime} \geq 0, \lambda_{n S}^{\prime}\left(S_{n}^{\max ^{2}}-u_{n P}^{* 2}-u_{n Q}^{* 2}\right)=0
$$

The Lagrangian function for the optimization problems (13) subject to (1)-(3) is specified as below

$$
\begin{aligned}
L\left(u_{s}, u_{n} ; \lambda, \mu\right)= & J(u)+\sum_{n=1}^{N} \lambda_{n P}\left(a_{n}-u_{n P}\right)+\sum_{n=1}^{N} \lambda_{n S}\left(S_{n}^{\max 2}-\left(u_{n P}^{2}+u_{n Q}^{2}\right)\right)+\mu_{P}\left(\sum_{n=1}^{N} u_{n P}-u_{s P}\right)+ \\
& \mu_{Q}\left(\sum_{n=1}^{N} u_{n Q}-u_{s Q}\right)
\end{aligned}
$$

(16)

The following, together with the equality constraint (5), are the Karush - Kuhn - Tucker (KKT) conditions for the constrained optimisation problems given in (13):

$$
\begin{aligned}
& v_{s P}^{\prime}\left(u_{s P}^{* *}\right)-\mu_{P}^{*} \leq 0, u_{s P}^{* *} \geq 0, u_{s P}^{* *}\left(v_{s P}^{\prime}\left(u_{s P}^{* *}\right)-\mu_{P}^{*}\right)=0 \\
& v_{s Q}^{\prime}\left(u_{s Q}^{* *}\right)-\mu_{Q}^{*} \leq 0, u_{s Q}^{* *} \geq 0, u_{s Q}^{* *}\left(v_{s Q}^{\prime}\left(u_{s Q}^{* *}\right)-\mu_{Q}^{*}\right)=0 \\
& g_{n P}^{\prime}\left(u_{n P}^{* *}\right)+2 \lambda_{n S}^{*} u_{n P}^{* *}+\lambda_{n P}^{*}-\mu_{P}^{*} \geq 0, u_{n P}^{* *} \geq 0, u_{n P}^{* *}\left(g_{n P}^{\prime}\left(u_{n P}^{* *}\right)+2 \lambda_{n S}^{*} u_{n P}^{* *}+\lambda_{n P}^{*}-\mu_{P}^{*}\right)=0 \\
& g_{n Q}^{\prime}\left(u_{n Q}^{* *}\right)+2 \lambda_{n S}^{*} u_{n Q}^{* *}-\mu_{Q}^{*} \geq 0, u_{n Q}^{* *} \geq 0, u_{n Q}^{* *}\left(g_{n Q}^{\prime}\left(u_{n Q}^{* *}\right)+2 \lambda_{n S}^{*} u_{n Q}^{* *}-\mu_{Q}^{*}\right)=0 \\
& u_{n P}^{* * 2}+u_{n Q}^{* * 2} \leq S_{n}^{\max ^{2}}, \lambda_{n S}^{*} \geq 0, \lambda_{n S}^{*}\left(S_{n}^{\max ^{2}}-u_{n P}^{* * 2}-u_{n Q}^{* * 2}\right)=0 \\
& u_{n P}^{* *} \leq a_{n}, \lambda_{n P}^{*} \geq 0, \lambda_{n P}^{*}\left(a_{n}-u_{n P}^{* *}\right)=0
\end{aligned}
$$

We should improve that (15) and (17) have the same solution.

Assuming (A1) and (A2), the underlying regulation problems are convex optimisation problems; Hence, the necessary KKT conditions are also sufficient for the optimality of the optimisation problems (12) and (13) (see in [16]). Thus, both of them have unique solution and they are characterised by their KKT conditions specified in (15) and (17) together with the equality constraint (5).

Then we can solve KKT functions (15) and get the $\boldsymbol{u}_{\boldsymbol{n}}$ expressions subject to $p_{P}$ and $p_{Q}$.

In case $p_{P}^{*}=0$ and $p_{Q}^{*} \geq 0$, we have $u_{n P}^{*}=0$ and $u_{n Q}^{*}=\left[g_{n Q}^{\prime}\left(p_{Q}^{*}\right)\right]^{-1} \leq S_{n}^{\max }$;

In case $p_{Q}^{*}=0$ and $0 \leq p_{P}^{*}<g_{n P}^{\prime}\left(u_{n P}^{\max }\right)$, we have $u_{n P}^{*}=\left[g_{n P}^{\prime}\left(p_{P}^{*}\right)\right]^{-1}<u_{n P}^{\max }, u_{n Q}^{*}=0$;

In case $p_{Q}^{*}=0$ and $p_{P}^{*} \geq g_{n P}^{\prime}\left(u_{n P}^{\max }\right)$, we have $u_{n P}^{*}=u_{n P}^{\max }, u_{n Q}^{*}=0$;

In case $0<p_{P}^{*}<g_{n P}^{\prime}\left(u_{n P}^{\max }\right)$ and $p_{Q}^{*}>0$, we have

(i) $u_{n P}^{*}=\left[g_{n P}^{\prime}\left(p_{P}^{*}\right)\right]^{-1}, u_{n Q}^{*}=\left[g_{n Q}^{\prime}\left(p_{Q}^{*}\right)\right]^{-1}$ if $u_{n P}^{* 2}+u_{n Q}^{* 2}<S_{n}^{\max ^{2}}$;

(ii) If the solution of (i) has constraint ${u_{n P}^{*}}^{2}+u_{n Q}^{*}{ }^{2}=S_{n}^{\max 2}$, we could get $\boldsymbol{u}_{n}$ from equations such that

$$
\left\{\begin{array}{l}
p_{P}^{*}-g_{n P}^{\prime}\left(u_{n P}^{*}\right)-2 \lambda_{n S}^{\prime} u_{n P}^{*}=0 \\
p_{Q}^{*}-g_{n Q}^{\prime}\left(u_{n Q}^{*}\right)-2 \lambda_{n S}^{\prime} u_{n Q}^{*}=0 \\
u_{n P}^{* 2}+u_{n Q}^{* 2}=S_{n}^{\max 2}
\end{array} ;\right.
$$

In case $p_{P}^{*} \geq g_{n P}^{\prime}\left(u_{n P}^{\max }\right)$ and $p_{Q}^{*}>0$, we have

(i) $u_{n P}^{*}=u_{n P}^{\max }$ and $u_{n Q}^{*}=\left[g_{n Q}^{\prime}\left(p_{Q}^{*}\right)\right]^{-1}$ if $u_{n P}^{* 2}+u_{n Q}^{* 2}<S_{n}^{\max ^{2}}$; 
(ii) $u_{n P}^{*}=u_{n P}^{\max }$ and $u_{n Q}^{*}=\sqrt{S_{n}^{\max ^{2}}-u_{n P}^{\max ^{2}}}$ if $u_{n P}^{* 2}+u_{n Q}^{* 2}=S_{n}^{\max 2}$.

The solutions of KKT conditions (17) are same to (15), lemma 1 is proved.

Before Lemma 2, we specify some notions below:

$$
\begin{aligned}
& \kappa_{P}=\inf \left\{\frac{1}{\varepsilon}\left[v_{s P}^{\prime}\left(u_{s P}+\varepsilon ; P\right)-v_{s P}^{\prime}\left(u_{s P} ; P\right)\right]\right\} \\
& v_{P}=\inf \left\{\inf \left\{\frac{1}{\varepsilon}\left[g_{n P}^{\prime}\left(u_{n P}+\varepsilon\right)-g_{n P}^{\prime}\left(u_{n P}\right)\right]\right\}\right\} \\
& \kappa_{Q}=\inf \left\{\frac{1}{\varepsilon}\left[v_{s Q}^{\prime}\left(u_{s Q}+\varepsilon ; Q\right)-v_{s Q}^{\prime}\left(u_{s Q} ; Q\right)\right]\right\} \\
& v_{Q}=\inf \left\{\inf \left\{\frac{1}{\varepsilon}\left[g_{n Q}^{\prime}\left(u_{n Q}+\varepsilon\right)-g_{n Q}^{\prime}\left(u_{n Q}\right)\right]\right\}\right\}
\end{aligned}
$$

Lemma 2. Suppose that $\eta_{P} \in\left(0, \frac{2 v_{P}}{v_{P}+N\left|\kappa_{P}\right|}\right)$ and $\eta_{Q} \in\left(0, \frac{2 v_{Q}}{v_{Q}+N\left|\kappa_{Q}\right|}\right)$, then the following asymptotic property holds:

$$
\lim _{k \rightarrow \infty} p^{(\mathrm{k})}=p^{*}
$$

Proof. To prove lemma 2 is equal to prove $\left|p_{P}^{*}-p_{P}^{(k+1)}\right|<\left|p_{P}^{*}-p_{P}^{(k)}\right|$ and $\left|p_{Q}^{*}-p_{Q}^{(k+1)}\right|<\left|p_{Q}^{*}-p_{Q}^{(k)}\right|$.

We prove inequations in case $p_{P}^{(k)}<p_{P}^{*}$ and $p_{Q}^{(k)}<p_{Q}^{*}$, in case $p_{P}^{(k)}<p_{P}^{*}$ and $p_{Q}^{(k)}>p_{Q}^{*}$, in case $p_{P}^{(k)}>p_{P}^{*}$ and $p_{Q}^{(k)}<p_{Q}^{*}$, in case $p_{P}^{(k)}>p_{P}^{*}$ and $p_{Q}^{(k)}>p_{Q}^{*}$. Here we take proof in one case.

In case $p_{P}^{(k)}<p_{P}^{*}$ and $p_{Q}^{(k)}<p_{Q}^{*}$.

According to allocation functions (15) and assuming (A1), (A2) we can donate parameters $\tau_{P}$ and $\tau_{Q}$ as follows

$$
\begin{aligned}
& v_{s P}^{\prime}\left(u_{s P}^{(k)} ; P\right)=\tau_{P}+p_{P}^{*} \\
& v_{s Q}^{\prime}\left(u_{s Q}^{(k)} ; Q\right)=\tau_{Q}+p_{Q}^{*}
\end{aligned}
$$

Together with (18), we have

$$
\begin{aligned}
& u_{n P}^{(k)} \geq u_{n P}^{*}-\frac{\psi_{P}}{v_{P}} \\
& u_{n Q}^{(k)} \geq u_{n Q}^{*}-\frac{\psi_{Q}}{v_{Q}}
\end{aligned}
$$

Donate $\psi_{P}=p_{P}^{*}-p_{P}^{(k)}$ and $\psi_{Q}=p_{Q}^{*}-p_{Q}^{(k)}$ and together with equation constraint (5), we can get

$$
u_{s P}^{*}-\frac{N \psi_{P}}{v_{P}} \leq u_{s P}^{(k)} \leq u_{s P}^{*}
$$


$u_{s Q}^{*}-\frac{N \psi_{Q}}{v_{Q}} \leq u_{s Q}^{(k)} \leq u_{s Q}^{*}$

According to the characteristic of convex function $v_{s P}\left(u_{s P} ; P\right)$, we have

$$
p_{P}^{*} \leq v_{s P}^{\prime}\left(u_{s P}^{(k)} ; P\right) \leq v_{s P}^{\prime}\left(u_{s P}^{*}-\frac{N \psi_{P}}{v_{P}} ; P\right)
$$

Together with (18), we can get

$$
p_{P}^{*} \leq v_{s P}^{\prime}\left(u_{s P}^{(k)} ; P\right) \leq p_{P}^{*}+\frac{N\left|\kappa_{P}\right|}{v_{P}} \psi_{P}
$$

Together with (19), we have

$$
\tau_{P} \leq \frac{N\left|\kappa_{P}\right|}{v_{P}} \psi_{P}, \quad \psi_{P}=p_{P}^{*}-p_{P}^{(k)}>0
$$

and obtain

$$
\tau_{Q} \leq \frac{N\left|\kappa_{Q}\right|}{v_{Q}} \psi_{Q}, \quad \psi_{Q}=p_{Q}^{*}-p_{Q}^{(k)}>0
$$

in the same way.

From (9), we have

$$
\begin{aligned}
& p_{P}^{(k+1)}-p_{P}^{(k)}=\eta_{P}\left(v_{s P}^{\prime}\left(u_{s P}^{(k)} ; P\right)-p_{P}^{(k)}\right) \\
& p_{Q}^{(k+1)}-p_{Q}^{(k)}=\eta_{Q}\left(v_{s Q}^{\prime}\left(u_{s Q}^{(k)} ; Q\right)-p_{Q}^{(k)}\right)
\end{aligned}
$$

Take (19) and (24) into (25) and together with target inequality, we can obtain the range of parameters as shown in lemma 2.

In other cases, we can get the same range of the parameters. Then lemma 2 is proved.

\section{Numerical examples}

In this section, we give numerical examples to verify our algorithm. We study the V2G coordination problems with $N=1000$, each of which possesses a battery with a capacity of $\Gamma_{n}=10 \mathrm{kWh}$, the maximum apparent power $S_{n}^{\max }=25 \mathrm{~kW}$ and the charging time $\Delta T=15 \mathrm{~min}$. More specifically, it is considered that the referred fixed active power $P=6 \mathrm{MW}$ and reactive power $Q=5 \mathrm{MVar}$ are required for the power grid.

As studied in [17], the initial SOC value of distribution vehicles approximately satisfies a Gaussian distribution. The initial SOC values are denoted by $\left\{S O C_{n 0} ; n \in \mathcal{N}\right\}$ and approximately satisfy a Gaussian distribution $N \sim\left(\mu, \delta^{2}\right)$, where $\mu=0.4$ and $\delta=0.1$. We also consider that $S O C_{\min }=10 \%$ and $S O C_{\max }=90 \%$.

Based upon the given specifications, we can specify the constraints of (1) - (3) for each individual PEV. We consider the cost functions of PEV $n$ as $g_{n P}\left(u_{n P}\right)=33 u_{n P}^{2} \sqrt{S O C_{n}}$ and $g_{n Q}\left(u_{n Q}\right)=30 u_{n Q}^{2} \sqrt{S O C_{n}}$, which are strictly convex and increasing. Then we consider the valuation function of the power grid as $v_{s P}\left(u_{s P}\right)=-0.1\left(u_{s P}-P\right)^{2}$ and $v_{s Q}\left(u_{s Q}\right)=-0.09\left(u_{s Q}-Q\right)^{2}$, which are strictly concave and increasing.

Under these data, we can calculate the range of price update parameters such that, $\eta_{P} \in(0,0.2)$ and $\eta_{Q} \in(0,0.2)$ 
solve the optimal problem shown as (13) and get the centralized results, i.e.

$\boldsymbol{u}_{s}^{* *}=\left(u_{s P}^{* *}, u_{s Q}^{* *}\right)=(5.30 \mathrm{MW}, 4.30 \mathrm{MVar})$

The decentralized results under price update mechanism are shown as follows

(i) case in $\eta_{P}=0.1, \quad \eta_{Q}=0.1$
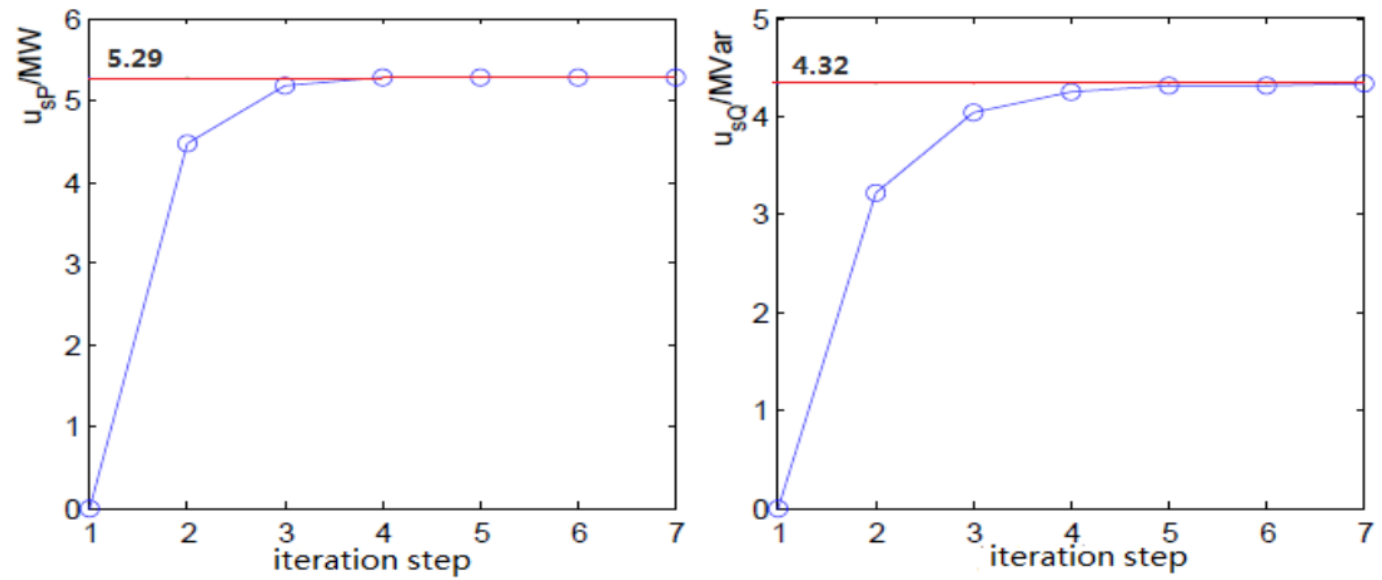

Figure 2 The allocation under price update mechanism when $\eta_{P}=0.1, \quad \eta_{Q}=0.1$

(ii) case in $\eta_{P}=0.2, \quad \eta_{Q}=0.2$
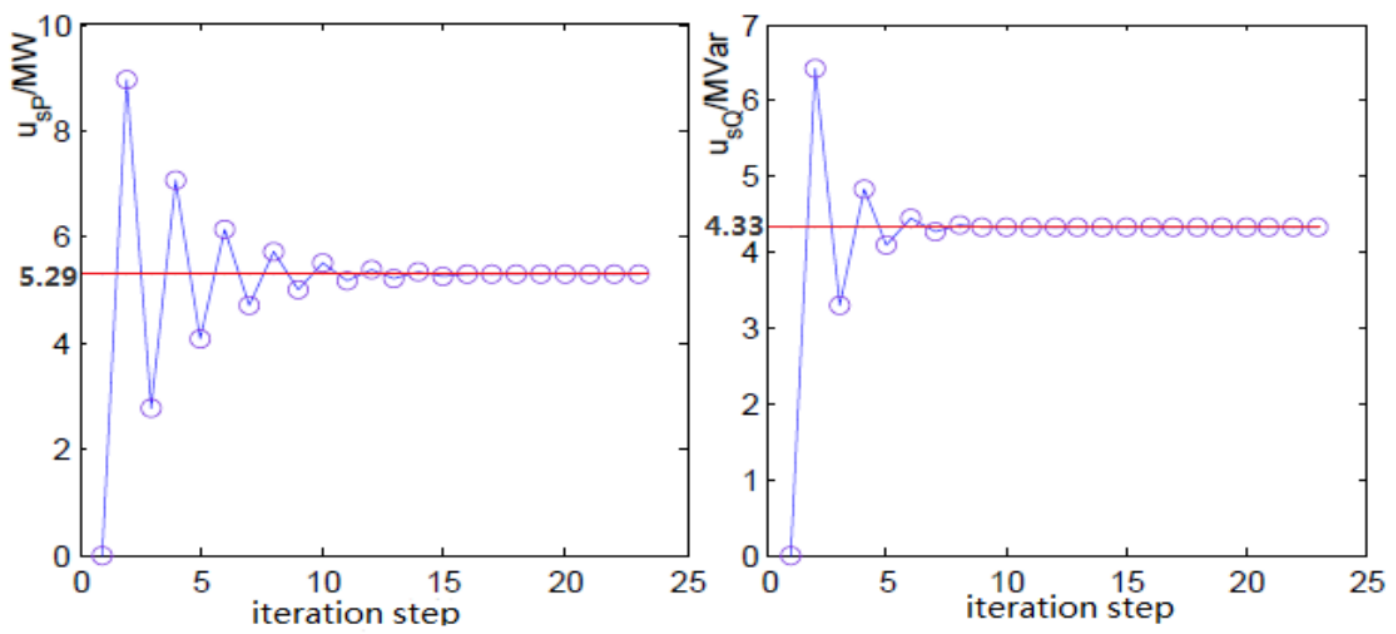

Figure 3 The allocation under price update mechanism when $\eta_{P}=0.2, \quad \eta_{Q}=0.2$

(iii) case in $\eta_{P}=0.4, \eta_{Q}=0.4$, we can't get the results because $\eta_{P}$ and $\eta_{Q}$ out of the range defined in lemma 2.

From this numerical example, we can verify lemma 1 and lemma 2 . When the price update parameters satisfy $\eta_{P} \in\left(0, \frac{2 v_{P}}{v_{P}+N\left|\kappa_{P}\right|}\right)$ and $\eta_{Q} \in\left(0, \frac{2 v_{Q}}{v_{Q}+N\left|\kappa_{Q}\right|}\right)$, the decentralized control allocations under price update mechanism could converge to centralized control results.

\section{Conclusion}

In this paper, we introduce a coordination problem of V2G to regulate the frequency and voltage and

develop a decentralized control method under price update mechanism. The solutions of this decentralized control method could converge to solutions of centralized control. 


\section{References}

[1] P. Denholm and W. Short, An evaluation of utility system impacts and benefits of optimally dispatched plug-in hybrid electric vehicles, National Renewable Energy Labortary, Technical Report NREL/TP-620-40293, October 2006.

[2] S. Rahman and G. Shrestha, An investigation into the impact of electric vehicle load on the electric utility distribution system, IEEE Transactions on Power Delivery, vol. 8, no. 2, pp. 591 - 597, 1993.

[3] F. Koyanagi and Y. Uriu, Modeling power consumption by electric vehicles and its impact on power demand, Electrical Engineering in Japan, vol. 120, no. 4, pp. 40 - 47, 1997.

[4] F. Koyanagi, T. Inuzuka, Y. Uriu, and R. Yokoyama, Monte Carlo simulation on the demand impact by quick chargers for electric vehicles, in IEEE Power Engineering Society Summer Meeting, vol. 2, Istanbul, Turkey, 18-22 July 1999, pp. 1031 - 1036.

[5] Wei Wei, Sha Qian, Han Yuankai, Li Jianxiang, Fu Chongguang. Impact analysis of electric vehicles charging on power grid. China Academic Journal Electronic Publishing House, 2011(6): 34-37.

[6] Z. Ma and D. Callaway, Centralized optimal charging of large population plug-in electric vehicles, Submitted to IEEE Transactions on Power Systems.

[7] Zhongjing Ma, D. Callaway, Inn Hiskens, Decentralized charging control for large populations of plug-in electric vehicles, Control Systems Technology, IEEE Transactions on, 1 - 12, Issue: 99, Nov. 2011.

[8] Zhongjing Ma, Long Ran. Optimal distributed charging coordination of plug-in electric vehicles with market uniform clearing mechanism. 25th Chinese Control and Decision Conference(CCDC), 2013: 3497-3502.

[9] Changsun Ahn, Chiao-Ting Li, Huei Peng, Optimal decentralized charging algorithm for electrified vehicles connected to smart grid, Journal of Power Sources, 10369C-10379, Vol.196, Issue.23.1, Dec. 2011.

[10] Pillai J R. Bak-Jensen B. Integration of vehicle-to-grid in the western Danish power system. IEEE Transactions on Sustainable Energy, 2011, 2(1): 12-19.

[11] Qiuwei Wu, Arne Hejde Nielsen, Jacob Østergaard, et, al. Potential analysis of regulating power from electric vehicle(EV) integration in Denmark. IEEE Power and Energy Society General Meeting, 2011: 153-177.

[12] Wu, C. Mohsenian-Rad, H. Huang, J. and Jatskevich, J. PEV based combined frequency and voltage regulation for smart grid. Innovative Smart Grid Technologies(ISGT), 2012: 1-6.

[13] Wu, C., Mohsenian-Rad, H., and Huang, J. Vehicle-to-aggregator interaction game. IEEE Transactions on Smart Grid, 2012, 3(1): 434-442.

[14] Xingyu Shi, Suli Zhou, Zhongjing Ma. A novel algorithm for divisible resource allocations under PSP auction mechanism. 26th Chinese Control and Decision Conference, 2014: 1723-1728.

[15] X. Shi and Z. Ma, An efficient game for vehicle-to-grid coordination problems in smart grid, International Journal of Systems Science, 2013.

[16] Boyd, S., \& Vandenberghe, L. Convex optimization. Cambridge: Cambridge University Press. 2004.

[17] Orr, J., \& Emanuel, A. Current harmonics generated by a cluster of electric vehicle battery chargers. IEEE Transactions on Power Apparatus and Systems, 1982,101(3): 691 - 700. 